\title{
Thermal fluctuations and electromagnetic noise spectra in quantum statistical mechanics.
}

\author{
Ladislaus Alexander Bányai*, Mircea Bundaru** \\ and Paul Gartner***
}

Institut für Theoretische Physik, Goethe-Universität, Frankfurt am Main* National Institute of Physics and Nuclear Engineering - Horia Hulubei, Bucharest**

National Institute of Materials Physics, POB MG-7, Bucharest-Magurele*** banyai@itp.uni-frankfurt.de, corresponding author

\begin{abstract}
We derive the thermal noise spectrum of the of the longitudinal and transverse electric field operator of a given wave vector starting from the quantum-statistical definitions and relate it to the complex frequency and wave vector dependent complex conductivity in a homogeneous, isotropic system of electromagnetic interacting electrons. No additional assumptions were used in the proof. We analyze separately the longitudinal and transverse case with their peculiarities. The Nyquist formula for vanishing frequency and wave vector, as well as its modification for non-vanishing frequencies and wave vectors follow immediately. Furthermore we discuss also the noise of the photon occupation numbers.
\end{abstract}

keywords: fluctuations, noise spectra, linear response, QED, longitudinal and transverse electric fields, Nyquist noise, photon number noise

\section{Introduction}

There are in the literature several theoretical approaches to the thermal noise in solids. The basic result is Nyquist's law from 1928 [1] for voltage noise at zero frequency, confirmed simultaneously by the experiment of Johnson [2]. Later in 1951 Callen and Welton [3] established a formula relating the integrated voltage noise spectrum to the frequency dependent resistance based on a fluctuationdissipation theorem. In 1985 Ref. [4] gave a derivation of the Callen-Welton spectral density based directly on the spectrum of the voltage fluctuations within a mean-field approximation, as well as a more exact one taking into account the Coulomb interactions. In this last formula the frequency dependent resistance is replaced by a certain frequency-dependent impedance. Thereafter, derivations 
of the noise spectral density under different stochastic, thermodynamic electric circuitry approaches with sometimes differing results were published and finally Ginzburg and Pitaevskii in 1988 [5] also confirmed the spectral density of CallenWelton within a Langevin approach to the electrical circuit, however only with constant resistance $\mathrm{R}$, inductance $\mathrm{L}$ and capacity $\mathrm{C}$.

Later review articles on the fluctuation-dissipation theorem [7] and noise [8], that are still mean-field theories and Ref.[8] concentrates more on mezoscopic systems.

The results of Ref. [4] went unmentioned by both Ref.[5] and [7],[8].

In a recent book of one of the authors [6] an alternative derivation of the old result of [4] was given, starting from the longitudinal electric field fluctuations. The extension of this approach is the aim of this paper. Within the frame of the non-relativistic QED we correlate the thermal noise spectral densities of longitudinal and transverse electric fields of a given wave vector $\mathbf{k}$ with the frequency and wave-vector dependent complex conductivity in a homogeneous, isotropic macroscopic system. We derive also an expression for the thermal noise spectral density of the photon occupation numbers of wave vector $\mathbf{k}$ that may be useful for mesoscopic systems.

One starts from the quantum-mechanical definition [9] of the fluctuation of an observable in a system in macro-canonical equilibrium defined as the average quadratic deviation in time. The noise spectrum is just its Fourier transform. We use the definition of the complex frequency and wave-vector dependent dielectric function (or conductivity) by the well-known modification of Kubo's linear response theory [10] in an e.m. interacting system due to [11] and [12]. As in [4] we stress the role of the internal electromagnetic fields (here including also the magnetic one) produced by the particles themselves. We treat ultimately the fluctuations of the local charge density and local current density as true sources of the electromagnetic equilibrium noise. We already have shown there that this leads to differences with respect to results obtained within the mean-field or the circuitry approaches, although quantitatively they may be not so important.

The only implicit thermodynamic element here is the expectation, that linear response theory is correct i.e. the thermodynamic limit introduces irreversibility.

Along this line we derive the thermal noise spectrum of a longitudinal electric field of wave-vector $\vec{k}$ in a homogeneous isotropic system. We obtain a generalized Nyquist formula relating it for non-vanishing frequency $\omega$ (of the noise) and wave-vector $\vec{k}$ (of the field) to the frequency and wave-vector dependent longitudinal dielectric function (or conductivity).

Thereafter, we derive the never touched noise spectrum of the transverse electric field (radiation field) starting from the non-relativistic QED by neglecting the retardation (i.e. in the $1 / c^{2}$ approximation). A Nyquist -like relation occurs again. Though, it has certain peculiarities due to the fact that unlike by the longitudinal field, the noise may be related to the conductivity only when the frequency $\omega$ of the noise is related to the wave vector of the field as $\omega=c k$.

Within the same $1 / c^{2}$ approximation of the QED we discuss also the noise 
spectrum of the photon occupation numbers. This also may be expressed through the electronic transverse current-current correlator. However due to the photonic factors under the integrals it cannot be related to the transverse conductivity.

Our results were obtained without supplementary assumptions.

We give also in the Appendices a comparison of this derivation to the alternative proof of the frequency dependent Nyquist formula given in Ref.[4] underlining the essential role of the charge density fluctuations in the longitudinal case. In this context we discuss the deficiency of the Callen-Welton fluctuationdissipation theorem [3] which was formulated years before the realization of the role of the internal field produced by the charged particles.

\section{Fluctuations and their spectral density.}

The time fluctuation $\Delta_{X}(t)$ of a given observable (hermitian operator) $X$ in thermal equilibrium is defined [9] as the average square deviation

$$
\Delta_{X}(t)=\left\langle(X(t)-X(0))^{2}\right\rangle_{0} \geq 0
$$

where the average is taken over the macro-canonical equilibrium density matrix

$$
\rho_{0} \equiv \frac{e^{-\beta(H-\mu N)}}{Z}
$$

and the time evolution of the operator $X$ is given by

$$
X(t)=e^{\frac{\imath H t}{\hbar}} X e^{\frac{-\imath H(t)}{\hbar}} .
$$

This is analogous to the general definition of a fluctuation both in classical- or quantum statistics.

Expanding Eq,1] one has

$$
\begin{aligned}
\Delta_{X}(t) & =\left\langle X(t)^{2}+X(0)^{2}-X(t) X(0)-X(0) X(t)\right\rangle_{0} \\
& =2\left\langle X(0)^{2}\right\rangle-\langle X(t) X(0)+X(0) X(t)\rangle_{0} .
\end{aligned}
$$

Leaving apart the constant $2\left\langle X(0)^{2}\right\rangle$, the time dependent entity of interest is

$$
\delta_{X}(t)=\langle X(t) X(0)+X(0) X(t)\rangle_{0}
$$

which is a real and even function of $t$. As a consequence its Fourier transform

$$
\tilde{\delta}_{X}(\omega)=\int_{-\infty}^{\infty} d t e^{\imath \omega t} \delta_{X}(t)=2 \int_{0}^{\infty} d t \cos (\omega t) \delta_{X}(t)
$$

is also real and even. Moreover, according to the Wiener-Khinchin Theorem [13, 14, 15] it is positive. This is defined [9] as the "noise" spectrum of $X$ in quantum statistics 1 .

\footnotetext{
${ }^{1}$ An asymmetrical "quantum noise" $\langle X(t) X\rangle_{0}$ as in Ref.[8] has no direct measurable physical meaning since it is not the average of a hermitian operator.
} 
It is easy to show, by expansion in the basis of the eigenfunctions of $H-\mu N$ for any observable $X$ (here $X \equiv X(0)$ ) the following two identities

$$
\begin{aligned}
\tilde{\delta}_{X}(\omega) & =2 \operatorname{coth}\left(\frac{\beta \hbar \omega}{2}\right) \Re\left\{\int_{0}^{\infty} d t e^{-\imath \omega t}\langle[X, X(t)]\rangle_{0}\right\} \\
& =2 \hbar \omega \operatorname{coth}\left(\frac{\beta \hbar \omega}{2}\right) \Re \int_{0}^{\infty} d t e^{-\imath \omega t} \int_{0}^{\beta} d \lambda\left\langle X X(t+\imath \hbar \lambda\rangle_{0} .\right.
\end{aligned}
$$

Correlators of both types appear in the linear response theory. Our purpose is to relate the electromagnetic thermal noise to the coefficients of that formalism.

\section{Linear response theory.}

\subsection{Linear response to an applied electromagnetic field.}

In a system of electromagnetic interacting charged particles the perturbing Hamiltonian contains only the applied (external) fields. However, the charged particles themselves are sources of electromagnetic fields. This makes the formulation of the problem more delicate. The linear part of the perturbation due to applied electromagnetic scalar $V^{\text {ext }}(\vec{x}, t)$ and vector potentials $\vec{A}^{\text {ext }}(\vec{x}, t)$ is

$$
H^{\prime}(t)=\int d \vec{x}\left\{\rho(\vec{x}) V^{e x t}(\vec{x}, t)-\vec{j}(\vec{x}) \vec{A}^{e x t}(\vec{x}, t)\right\}
$$

The average internal e.m. (electromagnetic) field has yet to be calculated. Together with the external field it gives rise to the total e.m. field in the system (matter). The conductivity is defined through the relationship between the average current and this total field. This relationship may be given explicitly only in homogeneous, isotropic systems after Fourier transformation in time and space variables.

The average of the current density operator

$$
\vec{j}(\vec{x}, t)=\frac{e}{2 m} \psi(\vec{x})^{+}\left(-\imath \hbar \nabla+\frac{e}{c} \vec{A}^{e x t}(\vec{x}, t)\right) \psi(\vec{x})+h . c
$$

given by the generalization ${ }^{2}$ of Kubo's adiabatic linear response theory [10] to the above general electromagnetic perturbation is

$$
\left\langle j_{\mu}(\vec{x}, t)\right\rangle=\lim _{s \rightarrow+0} \int_{-\infty}^{t} d t^{\prime} e^{s t^{\prime}} \int_{0}^{\beta} d \lambda \int d \vec{x}^{\prime}\left\langle j_{\nu}\left(\vec{x}^{\prime},-\imath \hbar \lambda\right) j_{\mu}\left(\vec{x}, t-t^{\prime}\right)\right\rangle_{0} E_{\nu}^{e x t}\left(\vec{x}^{\prime}, t^{\prime}\right)
$$

where $\mu, \nu=1,2,3$ are vector-indices and summation over double indices is understood, while $s$ is the adiabatic parameter we shall omit for simplicity in

\footnotetext{
${ }^{2}$ In his original paper Kubo considered only the perturbation by a constant homogeneous electric field.
} 
the following. (The current density operators in the correlator being already those in the absence of the external field!)

This relationship is gauge invariant and valid for any external electric field stemming from the e.m. potentials

$$
\vec{E}^{e x t}=-\nabla V^{e x t}-\frac{1}{c} \frac{\partial}{\partial t} \vec{A}^{e x t}
$$

It may be obtained by linear response to the perturbation Eq9, which involves its time-derivative, followed by the use of the continuity equation for $\partial \rho / \partial t$ and partial integration assuming the vanishing of the correlator at infinite spaceand time- distances.

If the e.m. interaction between the charged particles is ignored, one may reinterpret the e.m. field $\vec{E}^{e x t}$ as the mean-field $\vec{E}^{\text {mean }}$. Only within this approximation the kernel of Eq. 11 may be related directly to the conductivity of that system.

As it stays Eq.11 actually needs the formulation of the whole problem within the non-relativistic QED (see for example Ref.[6] for the definition of its Hamiltonian $H_{Q E D}$ ), not just the usual Coulomb Hamiltonian used in condensed matter theory. This emerges from the necessity to consider the averages of both the longitudinal and transverse electric fields.

However, after the first few steps we shall resort to a simplified approach by neglecting the retardation i.e. in an $1 / c^{2}$ approximation of the QED in which the motion of the electrons and photons are separated. The photons are considered to be free, while the charged particles interact directly by chargecharge and current-current forces. Their e.m. Hamiltonian [16, [17] (here for sake of simplicity just for electrons) is

$$
\begin{aligned}
H_{e . m .} & =\int d \vec{x} \psi^{+}(\vec{x})\left[\frac{\hbar^{2}}{2 m}\left(\nabla-\frac{\imath e}{\hbar c} \vec{A}^{e x t}(\vec{x}, t)\right)^{2}+e V^{e x t}(\vec{x}, t)\right] \psi(\vec{x}) \\
& +\frac{1}{2} \int d \vec{x} \int d \vec{x}^{\prime} \frac{N\left[\rho(\vec{x}) \rho\left(\vec{x}^{\prime}\right)-\frac{1}{c^{2}} \vec{j}_{T}(\vec{x}) \vec{j}_{T}\left(\vec{x}^{\prime}\right)\right]}{\left|\vec{x}-\vec{x}^{\prime}\right|}
\end{aligned}
$$

where $\vec{j}_{T}(\vec{x})$ is the transverse part of the current density Eq 10

$$
\vec{j}_{T}(\vec{r}, t) \equiv \vec{j}(\vec{r}, t)+\frac{1}{4 \pi} \nabla \int d \vec{r}^{\prime} \frac{\nabla^{\prime} \vec{j}\left(\vec{r}^{\prime}, t\right)}{\left|\vec{r}-\vec{r}^{\prime}\right|}
$$

and the symbol $N[\ldots]$ means taking the normal product of the operators.

Of course, more generally all charged particles (electrons and ions) should be included in the Hamiltonian, as well as their charge and current densities, but we limit here the discussion to the electron dynamics. 


\subsection{Frequency and wave vector dependent conductivity in a homogeneous, isotropic system.}

In a homogeneous (translation invariant) system one obtains by Fourier transformation in time and coordinate of Eq. 11

$$
\left\langle\tilde{j}_{\mu}(\vec{k}, \omega)\right\rangle=\int_{0}^{\infty} d t \int_{0}^{\beta} d \lambda \int d \vec{x} e^{\imath(\vec{k} \vec{x}+\omega t)}\left\langle j_{\nu}(0,0) j_{\mu}(\vec{x}, t+\imath \hbar \lambda)\right\rangle_{0} E_{\nu}^{e x t}(\omega, \vec{k})
$$

i.e. one has a local relationship in the Fourier space

$$
\left\langle\tilde{j}_{\mu}(\vec{k}, \omega)\right\rangle=\kappa(\vec{k}, \omega)_{\mu \nu} E_{\nu}^{e x t}(\omega, \vec{k})
$$

with

$$
\kappa(\vec{k}, \omega)_{\mu \nu}=\int_{0}^{\infty} d t \int_{0}^{\beta} d \lambda \int d \vec{x} e^{\imath(\vec{k} \vec{x}+\omega t)}\left\langle j_{\nu}(0,0) j_{\mu}(\vec{x}, t+\imath \hbar \lambda)\right\rangle_{0}
$$

If the system under consideration is isotropic, then one may separate the longitudinal and transverse parts

$$
\kappa(\vec{k}, \omega)_{\mu \nu}=\frac{k_{\mu} k_{\nu}}{k^{2}} \kappa_{L}(k, \omega)+\left(\delta_{\mu \nu}-\frac{k_{\mu} k_{\nu}}{k^{2}}\right) \kappa_{T}(k, \omega)
$$

and

$$
\begin{aligned}
& \left\langle\overrightarrow{\tilde{j}}_{L}(\vec{k}, \omega)\right\rangle=\kappa_{L}(k, \omega) \overrightarrow{\tilde{E}}_{L}^{\text {ext }}(\vec{k}, \omega) \\
& \left\langle\overrightarrow{\tilde{j}}_{T}(\vec{k}, \omega)\right\rangle=\kappa_{T}(k, \omega) \overrightarrow{\tilde{E}}_{T}^{\text {ext }}(\vec{k}, \omega) .
\end{aligned}
$$

It is important to remark here that the frequency and the wave vector in the transverse case are not independent $(\omega=c k)$.

\subsubsection{The longitudinal dielectric function and conductivity.}

In the longitudinal case it is convenient to consider the dielectric function $\epsilon(\vec{k}, \omega)$ using the relationship between the charge density and the scalar potential in the Coulomb gauge

$$
\langle\tilde{\rho}(\vec{k}, \omega)\rangle=\tilde{K}_{L}(k, \omega) \tilde{V}^{e x t}(\vec{k}, \omega)
$$

with

$$
\tilde{K}_{L}(k, \omega)=\int_{0}^{\infty} d t \int d \vec{x} e^{\imath \vec{k} \vec{x}} e^{\imath \omega t} K_{L}(\vec{x}, t)
$$

and

$$
K_{L}(\vec{x}, t) \equiv \frac{\theta(t)}{\imath \hbar}\langle[\rho(\vec{x}, t), \rho(0,0)]\rangle_{0}
$$

obtained directly from the linear response to the perturbation with the Hamiltonian of Eq. 9 in the presence of just a scalar external potential. 
Since in the Coulomb gauge the average internal and the external scalar potentials are given by the Poisson equations

$$
\begin{array}{r}
k^{2}\left\langle\tilde{V}^{i n t}(\vec{k}, \omega)\right\rangle=4 \pi\langle\tilde{\rho}(\vec{k}, \omega)\rangle \\
k^{2} \tilde{V}^{e x t}(\vec{k}, \omega)=4 \pi \tilde{\rho}^{e x t}(\vec{k}, \omega)
\end{array}
$$

and the longitudinal dielectric function is defined by the relationship between the external charge density and the total potential $V=V^{i n t}+V^{\text {ext }}$

$$
k^{2} \epsilon_{L}(k, \omega) \tilde{V}(\vec{k}, \omega)=4 \pi \tilde{\rho}^{e x t}(\vec{k}, \omega)
$$

by using Eq. 21 one gets

$$
\epsilon_{L}(k, \omega) \equiv \frac{1}{1+\frac{4 \pi}{k^{2}} \tilde{K}_{L}(\vec{k}, \omega)} .
$$

Using he continuity equation for the Fourier transforms of the averages

$$
\left\langle\tilde{j}_{L}(\vec{k}, \omega)\right\rangle=-\frac{\omega}{k}\langle\tilde{\rho}(\vec{k}, \omega)\rangle
$$

and $\tilde{E}_{L}^{e x t}(\vec{k}, \omega)=\imath k \tilde{V}^{e x t}(\vec{k}, \omega)$ one gets also

$$
\kappa_{L}(k, \omega)=-\frac{\imath \omega}{k^{2}} \tilde{K}_{L}(k, \omega) .
$$

Thus the complex longitudinal conductivity

$$
\sigma_{L}(k, \omega)=\frac{\kappa_{L}(k, \omega)}{1-\imath \frac{4 \pi}{\omega} \kappa_{L}(k, \omega)}
$$

results. The complex conductivity is of course related to the complex dielectric function by

$$
\epsilon_{L}(k, \omega)=1-\frac{4 \pi}{\imath \omega} \sigma_{L}(k, \omega)
$$

It is important to have in mind that in the mean-field approximation that considers Coulomb forces only in a self-consistent manner, the longitudinal conductivity is just proportional to the longitudinal current correlator of the noninteracting charges.

\subsubsection{The transverse conductivity.}

From

$$
\left\langle\overrightarrow{\tilde{j}}_{T}(\vec{k}, \omega)\right\rangle=\kappa_{T}(k, \omega) \overrightarrow{\tilde{E}}_{T}^{e x t}(\vec{k}, \omega)
$$

neglecting the retardation

$$
\left\langle\overrightarrow{\tilde{E}}_{T}^{i n t}(\vec{k}, \omega)\right\rangle=\imath \omega \frac{4 \pi}{c^{2} k^{2}}\left\langle\overrightarrow{\tilde{j}}_{T}(\vec{k}, \omega)\right\rangle=\imath \omega \frac{4 \pi}{c^{2} k^{2}} \kappa_{T}(k, \omega) \overrightarrow{\tilde{E}}_{T}^{\text {ext }}(\vec{k}, \omega)
$$


and therefore with the definition

$$
\left\langle\overrightarrow{\tilde{j}}_{T}(\vec{k}, \omega)\right\rangle=\sigma_{T}(k, \omega)\left(\overrightarrow{\tilde{E}}_{T}^{e x t}(\vec{k}, \omega)+\left\langle\overrightarrow{\tilde{E}}_{T}^{i n t}(\vec{k}, \omega)\right\rangle\right)
$$

we get

$$
\sigma_{T}(k, \omega)=\frac{\kappa_{T}(k, \omega)}{1+\imath \omega \frac{4 \pi}{c^{2} k^{2}} \kappa_{T}(k, \omega)}
$$

Since the transverse external electric field obeys the wave equation, in these equations the variables $\omega$ and $k$ are related by $\omega=c k$ ! Therefore one might speak just about a frequency dependent transverse conductivity

$$
\sigma_{T}(\omega)=\frac{\kappa_{T}(\omega / c, \omega)}{1+\imath \frac{4 \pi}{\omega} \kappa_{T}(\omega / c, \omega)}
$$

\subsection{The noise spectrum of the longitudinal electric field.}

From the hermiticity of the operator $\vec{E}(\vec{x}, t)$ it follows that after Fourier transforming in the space variable obeys $\overrightarrow{\tilde{E}}(-\vec{k}, t)^{+}=\overrightarrow{\tilde{E}}(\vec{k}, t)$ and we may define two hermitian scalar operators (observables) as the "real" and "imaginary" parts of the longitudinal field operator $\frac{\vec{k}}{k} \vec{E}(\vec{k}, t)$

$$
\begin{aligned}
& E_{L}^{R}(\vec{k}, t)=\frac{\vec{k}}{2 k}(\overrightarrow{\tilde{E}}(\vec{k}, t)+\overrightarrow{\tilde{E}}(-\vec{k}, t)) \\
& E_{L}^{I}(\vec{k}, t)=-\imath \frac{\vec{k}}{2 k}(\overrightarrow{\tilde{E}}(\vec{k}, t)-\overrightarrow{\tilde{E}}(-\vec{k}, t))
\end{aligned}
$$

The noise of any of these observables (omitting the upper indices R,I) according Eq. 7 is

$$
\tilde{\delta}_{E_{L}}(\omega, k)=2 \operatorname{coth}\left(\frac{\beta \hbar \omega}{2}\right) \Re\left\{\int_{0}^{\infty} d t e^{-\imath \omega t}\left\langle\left[E_{L}(\vec{k}, 0), E_{L}(\vec{k}, t)\right]\right\rangle_{0}\right\}
$$

Here the inverted order of arguments $(\omega, k)$ in the notation underlines that $\vec{k}$ is the wave-vector of the electric field, while $\omega$ is the frequency of the noise spectrum.

The Fourier transform of the longitudinal electric field is related by the Poisson equation $(\nabla \vec{E}(\vec{x}, t)=4 \pi \rho(\vec{x}, t))$ to the Fourier transform of the charge density by

$$
\tilde{E}_{L}(\vec{k}, t)=\imath \frac{4 \pi}{k} \tilde{\rho}(\vec{k}, t) .
$$

It is obvious that the quantities of interest are $\langle[\tilde{\rho}(\vec{k}), \tilde{\rho}(\vec{q}, t)]\rangle$ with $\vec{q}= \pm \vec{k}$. One has, using translation invariance in homogeneous systems 


$$
\begin{aligned}
\langle[\tilde{\rho}(\vec{k}), \tilde{\rho}(\vec{q}, t)]\rangle_{0} & =\int d \vec{x}^{\prime} e^{\imath \vec{q} \vec{x}^{\prime}} \int d \vec{x} e^{\imath \vec{k} \vec{x}}\left\langle\left[\rho\left(\vec{x}-\vec{x}^{\prime}\right), \rho(0, t)\right]\right\rangle_{0} \\
& \left.\left.=\int d \vec{x}^{\prime} e^{\imath(\vec{k}+\vec{q}) \vec{x}^{\prime}} \int d \vec{x} e^{\imath \vec{k} \vec{x}},\right\rangle[\rho(\vec{x}), \rho(0, t)]\right\rangle_{0} \\
& =\Omega \delta_{\vec{k}+\vec{q}, 0} \int d \vec{x} e^{2 \vec{k} \vec{x}}\langle[\rho(\vec{x}), \rho(0, t)]\rangle_{0}
\end{aligned}
$$

with $\Omega$ being the volume (under cyclic boundary conditions).

In the case $\vec{q}=\vec{k}$ the $\delta$-condition implies $\vec{k}=0$, and one is left with the commutator of the total charge with the charge density at the origin. The former is a conserved quantity and therefore it can be taken at any time, in particular at the same time $t$ as the local charge, whith the result that their commutator is zero. Therefore only the case $\vec{q}=-\vec{k}$ contributes, and one gets

$$
\begin{aligned}
& \tilde{\delta}_{E_{L}}(\omega, k)= \\
& -2 \Omega \operatorname{coth}\left(\frac{\beta \hbar \omega}{2}\right) \frac{(4 \pi)^{2}}{k^{2}} \Re\left\{\int_{0}^{\infty} d t e^{-\imath \omega t} \int d \vec{x} e^{-\imath \vec{k} \vec{x}}\langle[\rho(\vec{x}), \rho(0, t)]\rangle_{0}\right\} .
\end{aligned}
$$

On the other hand, for Coulomb systems in the frame of the linear response theory (see Eqs. 22, 23 and 27) the following relationship for the longitudinal dielectric function holds

$$
\frac{4 \pi}{\hbar k^{2}} \Re\left\{\int_{0}^{\infty} d t e^{-\imath \omega t} \int d \vec{x} e^{-\imath \vec{k} \vec{x}}\langle[\rho(\vec{x}), \rho(0, t)]\rangle_{0}\right\}=\Im\left\{\frac{1}{\epsilon_{L}(\vec{k}, \omega)}\right\}
$$

and we identify

$$
\tilde{\delta}_{E_{L}}(\omega, k)=-8 \pi \hbar \Omega \operatorname{coth}\left(\frac{\beta \hbar \omega}{2}\right) \Im\left\{\frac{1}{\epsilon_{L}(\vec{k}, \omega)}\right\} .
$$

This is our most general new result for the noise spectrum of the longitudinal electric field of wave vector $\vec{k}$.

In the classical limit $\hbar \rightarrow 0$ it reduces to

$$
\lim _{\hbar \rightarrow 0} \tilde{\delta}_{E_{L}}(\omega, k)=-\frac{16 \pi \Omega}{\beta \omega} \Im\left\{\frac{1}{\epsilon_{L}(\vec{k}, \omega)}\right\} .
$$

This coincides with the corresponding result [18] obtained in the frame of the classical plasma theory.

In the quantum mechanical version for $\vec{k} \rightarrow 0$ one gets

$$
\tilde{\delta}_{E_{L}}(\omega, 0)=-8 \pi \hbar \Omega \operatorname{coth}\left(\frac{\beta \hbar \omega}{2}\right) \Im\left\{\frac{1}{\epsilon_{L}(0, \omega)}\right\} .
$$

This last result coincides (up to slight differences in the definitions and unit systems) with those obtained earlier in Ref. [4] and [6]. 
Its extensive formulation with a sample of length $\mathrm{L}$ and cross-section $\mathrm{S}$ gives rise for the noise of the voltage drop $U(\omega) \equiv L \frac{1}{\Omega} \int d \mathbf{x} \tilde{E}_{L}(\mathbf{x}, \omega)=\frac{1}{S} \tilde{E}_{L}(\omega, 0)$ a slightly modified Callen-Welton noise spectral density ${ }^{3}$

$$
\tilde{\delta}_{U}(\omega, 0)=2 \hbar \omega \operatorname{coth}\left(\frac{\beta \hbar \omega}{2}\right) \Re Z(\omega) .
$$

Here $Z(\omega)$ represents the impedance

$$
\frac{1}{Z(\omega)}=\frac{1}{R(\omega)}+\imath \omega C(\omega)
$$

of parallel linked resistance

$$
R(\omega)=\frac{L}{S \Re \sigma_{L}(\omega)}
$$

with with $\Re \sigma_{L}(\omega) \equiv \frac{\omega}{4 \pi} \Im \epsilon(\omega)$ according to Eq. 31 and capacity between the end cross-sections of the sample

$$
C(\omega)=\frac{S}{L 4 \pi} \Re \epsilon_{L}(\omega) .
$$

In the $\omega \rightarrow 0$ limit, both the resistance and the capacity remain finite, thus the contribution of the capacity to the impedance vanishes. As a result, in this limit the old Nyquist theorem [1]

$$
\tilde{\delta}_{U}(0,0)=4 R k_{B} T
$$

emerges.

In the mean-field version the capacity $C(\omega)$ that stems from the explicit Coulomb interaction is absent and one gets the standard Callen-Welton spectral density. This occurs implicitly if $(\omega C(\omega) R(\omega))^{2} \ll 1.4$

\section{The noise spectrum of the transverse electric field.}

Let us consider now the noise of the transverse (radiation) electric field $\vec{E}_{T}(\vec{x}, t)$ in a homogeneous, isotropic sample. There are two possible polarizations of this field defined by the two orthogonal to each other and to the wave vector $\vec{k}$ unit vectors $\vec{e}_{\vec{k}}^{(\lambda)}=\vec{e}_{-\vec{k}}^{(\lambda)}(\lambda=1,2)$.

The transverse electric field operator in the non-relativistic QED is given by

$$
\vec{E}_{T}(\vec{x}, t)=-\frac{1}{c} \frac{\partial}{\partial t} \vec{A}_{T}(\vec{x}, t)
$$

\footnotetext{
${ }^{3}$ In [4] unfortunately a misprint occurred (namely instead of $\Re Z(\omega)$ stand $\Re Z(\omega)^{-1}$ )!

${ }^{4}$ Actually Johnson[2] fitted his original experiment with the classical $(\hbar \rightarrow 0)$ version of the integrated voltage noise formula of Nyquist[1] while including the capacity effect proven later in Ref.[4].
} 
with the transverse vector potential operator (radiation field) in the Heisenberg image of the free Hamiltonian being

$$
\vec{A}_{T}(\vec{x}, t)=\sqrt{\frac{\hbar c}{\Omega}} \sum_{\lambda=1,2} \sum_{\vec{q}} \frac{1}{\sqrt{|\vec{q}|}} \vec{e}_{\vec{q}}^{(\lambda)} e^{-\imath \vec{q} \vec{x}}\left(e^{-\imath c q t} b_{\vec{q}, \lambda}+e^{\imath c q t} b_{-\vec{q}, \lambda}^{+}\right)
$$

(with periodical boundary conditions) and

$$
\left[b_{\vec{q}, \lambda}, b_{\vec{q}^{\prime}, \lambda^{\prime}}^{+}\right]=\delta_{\vec{q}, \vec{q}^{\prime}} \delta_{\lambda, \lambda^{\prime}}
$$

We consider analogously to the treatment of the noise of the longitudinal electric field the two hermitian (scalar) operators constructed from the Fourier transform of the electrical field in the space variable

$$
\begin{aligned}
& E_{T}^{R}(\vec{k}, t)=\frac{1}{2} \vec{e}_{\vec{k}}\left(\overrightarrow{\tilde{E}}_{T}(\vec{k}, t)+\overrightarrow{\tilde{E}}_{T}(-\vec{k}, t)\right) \\
& E_{T}^{I}(\vec{k}, t)=-\imath \frac{1}{2} \vec{e}_{\vec{k}}\left(\overrightarrow{\tilde{E}}_{T}(\vec{k}, t)-\overrightarrow{\tilde{E}}_{T}(-\vec{k}, t)\right)
\end{aligned}
$$

for an arbitrary polarization direction $\vec{e}_{\vec{k}}$.

The noise of the observable $E_{T}^{R}$ according to Eq.8 is

$$
\tilde{\delta}_{E_{T}^{R}}(\omega, k)=2 \hbar \omega \operatorname{coth}\left(\frac{\beta \hbar \omega}{2}\right) \Re \int_{0}^{\infty} d t e^{-\imath \omega t} \int_{0}^{\beta} d \lambda\left\langle E_{T}^{R}(\vec{k}, 0) E_{T}^{R}(\vec{k}, t+\imath \hbar \lambda)\right\rangle_{0}
$$

and analogously for $E_{T}^{I}$.

From this step on we shall neglect the retardation. Then since only the internal electric field fluctuates one may express the transverse electric field directly by the transverse current density

$$
E_{T}(\vec{k}, t)=-\frac{4 \pi}{(c k)^{2}} \frac{\partial}{\partial t} j_{T}(\vec{k}, t)
$$

where of course $j_{T}(\vec{k}, t)$ is the projection of the current density on the same chosen polarization direction. Consequently we consider the time evolution also in the $1 / c^{2}$ approximation i.e. in the frame of the Hamiltonian Eq. 13.

Thereafter we get, as in the longitudinal case the only surviving contribution

$$
\tilde{\delta}_{E_{T}}(\omega, k)=\frac{(4 \pi)^{2}}{(c k)^{4}} \hbar \omega \operatorname{coth}\left(\frac{\beta \hbar \omega}{2}\right) \Re \int_{0}^{\infty} d t e^{-\imath \omega t} \int_{0}^{\beta} d \lambda\left\langle\dot{j}_{T}(\vec{k}, 0) \dot{j}_{T}(-\vec{k}, t+\imath \hbar \lambda)\right\rangle_{0} .
$$

(Since this equation is valid for both fields $E_{T}^{R}$ and $E_{T}^{I}$ we omited the $R, I$ indices.) However, here the argument for the absence of the other contributions is due only to the presence of the Kronecker-delta $\delta_{\vec{k}}$ in front of them. For our final result relating the noise spectrum to the transverse conductivity these are anyway irrelevant since unlike in the longitudinal case, the wave vector and the frequency of the transverse field are not independent 
Due to time translation invariance of the equilibrium average one may transfer the time derivative of the first current on the second one (implying a change of sign) and after two integration by parts one has

$$
\begin{gathered}
\int_{0}^{\infty} d t e^{-\imath \omega t} \int_{0}^{\beta} d \lambda\left\langle\dot{j}_{T}(\vec{k}, 0) \dot{j}_{T}(-\vec{k}, t+\imath \hbar \lambda)\right\rangle_{0}= \\
\omega^{2} \int_{0}^{\infty} d t e^{-\imath \omega t} \int_{0}^{\beta} d \lambda\left\langle j_{T}(\vec{k}, 0) j_{T}(-\vec{k}, t+\imath \hbar \lambda)\right\rangle_{0} .
\end{gathered}
$$

The integrated parts do not contribute, and the simplest way to see this is by recalling that fluctuations were originally expressed as a time integral over the whole real axis, see Eq, 6 , and the correlations are supposed to vanish at $\pm \infty$. Alternatively, a direct proof is obtained by using the invariance against space inversion to show that the real part of the integral over $\lambda$ is even in time, while the imaginary part is odd. As a consequence, the time integration may be extended to $-\infty$.

Further,

$$
\left\langle j_{T}(\vec{k}, 0) j_{T}(-\vec{k}, t+\imath \hbar \lambda)\right\rangle_{0}=\Omega \int d \vec{x} e^{\imath \vec{k} \vec{x}}\left\langle j_{T}(0,0) j_{T}(\vec{x}, t+\imath \hbar \lambda)\right\rangle_{0}
$$

and therefore we have finally

$$
\begin{aligned}
\tilde{\delta}_{E_{T}}(\omega, k) & =\Omega \omega^{2} \frac{(4 \pi)^{2}}{(c k)^{4}} \hbar \omega \operatorname{coth}\left(\frac{\beta \hbar \omega}{2}\right) \\
& \times \Re \int_{0}^{\infty} d t e^{-\imath \omega t} \int_{0}^{\beta} d \lambda \int d \vec{x} e^{\imath \vec{k} \vec{x}}\left\langle j_{T}(0,0) j_{T}(\vec{x}, t+\imath \hbar \lambda)\right\rangle_{0} .
\end{aligned}
$$

Now, from Eq, 17 we had

$$
\kappa(k, \omega)_{T}=\int_{0}^{\infty} d t \int_{0}^{\beta} d \lambda \int d \vec{x} e^{\imath(\vec{k} \vec{x}+\omega t)}\left\langle j_{T}(0,0) j_{T}(\vec{x}, t+\imath \hbar \lambda)\right\rangle_{0}
$$

and using Eq 35

$$
\kappa(k, \omega)_{T}=\frac{\sigma_{T}(\omega, k)}{1-\imath \omega \frac{4 \pi}{c^{2} k^{2}} \sigma_{T}(k, \omega)} .
$$

At first glance it seems that we found a simple relationship between the noise of the transverse field and the transverse conductivity. However the correct interpretation is more subtle. In the case of the transverse conductivity the variables $\omega$ and $k$ are not independent: $\omega=c k$. Therefore, according to Eq. 36 one has just a frequency dependent conductivity and

$$
\kappa(\omega, \omega / c)_{T}=\frac{\sigma_{T}(\omega)}{1-\frac{4 \pi \imath}{\omega} \sigma_{T}(\omega)} .
$$

In the transverse noise spectral density $k$ is the wave vector of the electric field while $\omega$ is the frequency of the noise. They are independent variables. A 
direct relationship to the transverse conductivity however exists only along the line $\omega=c k$.

Thus

$$
\left.\tilde{\delta}_{E_{T}}(\omega, k)\right|_{k=\omega / c}=\Omega 4 \pi \hbar \operatorname{coth}\left(\frac{\beta \hbar \omega}{2}\right) \Im \frac{1}{1-\frac{4 \pi \imath}{\omega} \sigma_{T}(\omega)} .
$$

This relation is the analogue of Eq 44 for the noise spectrum of longitudinal fields.

Actually our definition and discussion of the noise spectrum of the electric field follows that of Ref.[6] including now the dependence on the wave vector $\mathbf{k}$ and brings it farther through the $1 / c^{2}$ approximation for the transverse conductivity.

From Eq.44, Eq 66 and the positivity of the noise spectral density follows also the positivity of the real part of the longitudinal/transversal conductivity $(\Re \sigma(\omega, k) \geq 0)$ within the frame of the linear response theory.

\section{The noise spectrum of the photon occupation numbers.}

Beside the field noises one might consider also the noise of the photon occupation numbers. The noise spectral density of the photon occupation numbers $n_{\vec{k}}$ (with an arbitrary chosen polarization not mentioned) is given by

$$
\tilde{\delta}_{n_{\vec{k}}}(\omega)=2 \hbar \omega \operatorname{coth}\left(\frac{\beta \hbar \omega}{2}\right) \Re \int_{0}^{\infty} d t e^{-\imath \omega t} \int_{0}^{\beta} d \lambda\left\langle\hat{n}_{\vec{k}} \hat{n}_{\vec{k}}(t+\imath \hbar \lambda\rangle_{0}\right.
$$

where $\hat{n}_{\vec{k}} \equiv b_{\vec{k}}^{+} b_{\vec{k}}$ is the operator of the number of photons of wave vector $\vec{k}$.

On the other hand analogously to Eq60 we have

$$
\int_{0}^{\infty} d t e^{-\imath \omega t}\left\langle\hat{n}_{\vec{k}}(0) \hat{n}_{\vec{k}}(t+\imath \hbar \lambda)\right\rangle_{0}=\frac{1}{\omega^{2}} \int_{0}^{\infty} d t e^{-\imath \omega t}\left\langle\dot{\hat{n}}_{\vec{k}}(0) \dot{\hat{n}}_{\vec{k}}(t+\imath \hbar \lambda)\right\rangle_{0} .
$$

However, retaining the lowest order in $\frac{1}{c}$ from the full QED Hamiltonian

$$
\frac{1}{c} \vec{j}_{T}(\vec{x}, t) \vec{A}_{T}
$$

we have

$$
\dot{n}_{\vec{k}}(t)=\frac{\imath}{\hbar c} \int d \vec{x}\left[\vec{j}_{T}(\vec{x}, t) \vec{A}_{T}(\vec{x}, t), n_{\vec{k}}(t)\right]
$$

and

$$
\begin{aligned}
& \int_{0}^{\infty} d t e^{-\imath \omega t}\left\langle n_{\vec{k}}(0) n_{\vec{k}}(t+\imath \hbar \lambda)\right\rangle_{0}=-\frac{1}{(\hbar \omega c)^{2}} \int_{0}^{\infty} d t e^{-\imath \omega t} \int d \vec{x} \int d \vec{x}^{\prime} \\
& \left\langle\left[\vec{j}_{T}(\vec{x}, 0) \vec{A}_{T}(\vec{x}, 0), n_{\vec{k}}(0)\right]\left[\vec{j}_{T}\left(\vec{x}^{\prime}, t+\imath \hbar \lambda\right) \vec{A}_{T}\left(\vec{x}^{\prime}, t+\imath \hbar \lambda\right), n_{\vec{k}}(t+\imath \hbar \lambda)\right]\right\rangle_{0}
\end{aligned}
$$

where the transverse vector potential operator is defined by Eq. 53 . 
In what follows we neglect as before the retardation and ignore consequently terms higher order than $1 / c^{2}$. Within this approximation we remain only with an approximate Hamiltonian being the sum of the $\frac{1}{c^{2}}$ e.m Hamiltonian Eq. 13 (as well as some other interactions) for electrons and of the free Hamiltonian of the photons both in the averaging over equilibrium as in the time evolution.

Performing the commutations and taking again into account the translation, rotation and reflection invariance in the coordinate space we get after some algebra

$$
\begin{aligned}
& \tilde{\delta}_{n_{\vec{k}}}(\omega)=2 \frac{\operatorname{coth}\left(\frac{\beta \hbar \omega}{2}\right)}{c k \omega} \Re \int_{0}^{\infty} d t e^{-\imath \omega t} \int_{0}^{\beta} d \lambda\left\langle\tilde{j}(\vec{k}, 0) j_{T}(0, t+\imath \hbar \lambda)\right\rangle_{0}^{e l} \times \\
& {\left[e^{-\imath(\omega-c k) t-c k \lambda}\left(1+\mathcal{N}_{k}\right)+e^{-\imath(\omega+c k) t+c k \lambda)} \mathcal{N}_{k}\right]}
\end{aligned}
$$

where where the second current operator is $\left.j(\mathbf{x}, t+\imath \hbar \lambda)\right|_{\mathbf{x}=0}$ and

$$
\mathcal{N}_{k} \equiv \frac{1}{e^{\beta k c}-1}
$$

is the Bose distribution of photons.

The above expression resembles Eq 62 for the noise of the transverse electric field, however they differ essentially due to the presence of the photonic factors alongside the electronic current-current correlator under the integrals. Therefore, it cannot be related to the transverse conductivity. Nevertheless, Eq. 72 may be the starting point for the direct computation of the photon number noise spectrum within some approximations for homogeneous, isotropic solid state models.

On the other hand, photon number fluctuations may be very important in mesoscopical systems in the "quantum limit". The above equation, whose derivation does not depend on the thermodynamic limit may serve for their discussion.

\section{Conclusions.}

We started from the generally accepted quantum statistical definition [9] of the thermal fluctuation of an observable as its average square deviation in time and the noise as its Fourier transform. We have chosen, within the frame of the nonrelativistic QED, as relevant electromagnetic observables the real and imaginary (hermitian) parts of the Fourier transforms of the longitudinal $\tilde{E}_{L}(t, \vec{k})$ and transverse $\tilde{E}_{T}(t, \vec{k})$ electric fields in a homogeneous isotropic system.

We use throughout the improved linear response theory according to the proper definition of the total field in a system of electromagnetically interacting charged particles according to [11] and [12] (see also Ref.[6] ) to relate the thermal noise spectrum of the above operators to the frequency and wave-vector dependent complex dielectric function (or conductivity).

Our result in the longitudinal case is just the the quantum-mechanical version of the old analysis of electric fluctuations [18] in the classical plasma theory. 
In the zero wave vector and zero frequency limit the classical Nyquist theorem [1] results, while for non-vanishing frequencies a modification of the result of Ref.[3] as it was predicted earlier in Ref.[4] emerges.

Actually we got as well in the longitudinal as in the transverse case more general results Eq.44 and Eq.66, including $k \neq 0$. However, in the transverse case the relationship to the transverse conductivity occurs obviously only for $\omega=c k$ and it was obtained from the QED within the $1 / c^{2}$ approximation i.e. after neglecting the retardation. These relations may be looked at as generalized Nyquist theorems for the noise spectra of intensive entities.

Finally, we discussed also the noise spectrum of the photon occupation numbers within the same $1 / c^{2}$ approximation. This noise spectrum Eq.72 is again related to the transverse electronic current-current correlator. However, due to the presence of the Bose functions under the integrals it cannot be related to the transverse conductivity. Nevertheless, this result may be useful for mesoscopic systems.

These three kind of noise spectra we discussed exhaust the experimental configurations of electromagnetic thermal fluctuations in (macroscopic) condensed matter.

It is important to mention, that in our derivations no other ingredients were introduced except the implicit assumption of linear response theory about the time-decay of correlations (i.e. irreversibility in the thermodynamic limit).

\section{Appendices}

\section{A The original derivation of Ref [4].}

The result of Section 3.3 for the spectral density of the thermal noise Eq46 was earlier derived by Ref.[4], however in a different manner. One considers a sample of length $L$ (along the $z$ axis) and cross-section $S$ and defines the voltage drop along the sample as

$$
U=\frac{1}{S} \int d x d y[V(x, y, L)-V(x, y, 0)]
$$

wher 5

$$
V(\vec{x})=\int d \vec{x} \frac{\rho\left(\vec{x}^{\prime}\right)}{\left|\vec{x}-\vec{x}^{\prime}\right|}
$$

is the potential created by the internal charge density. In the limit $S \rightarrow \infty$ one has

$$
U=\frac{4 \pi}{S} \int d \vec{x} z \rho(\vec{x})
$$

\footnotetext{
${ }^{5}$ In this paper we use the Gauss system of units, while in [4] the SI system was used and the sign of the frequency $\omega$ in the linear response was opposite to the present choice.
} 
Using the equation of continuity

$$
\frac{\partial \rho(\vec{x}, t)}{\partial t}+\nabla \vec{j}(\vec{x}, t)=0
$$

and the vanishing of the normal component of the current density at the ends of the sample (isolated sample!) one relates the potential drop to the averaged charge flux in the $z$-direction

$$
I \equiv \frac{S}{\Omega} \int d \vec{x} j_{z}(\vec{x})
$$

by

$$
\frac{\partial U(t)}{\partial t}=4 \pi \frac{L}{S} I(t)
$$

and correspondingly the spectral densities of their fluctuations (with the original notations) are related

$$
\delta U_{\omega}^{2}=\left(\frac{4 \pi L}{S \omega}\right)^{2} \delta I_{\omega}^{2} .
$$

On the other hand, using linear response theory for the average current $I$ and the noise formula for $\delta I_{\omega}^{2}$, as well as taking into account also the relationship between the complex conductivity and the complex dielectric function one gets (see Eq. 2.11 of Ref.[4])

$$
\delta U_{\omega}^{2}=2 \hbar \omega \operatorname{coth}\left(\frac{\beta \hbar \omega}{2}\right) R(\omega) \frac{1}{1+\left(\frac{\omega \Re \epsilon(\omega)}{\Re \sigma(\omega)}\right)^{2}} .
$$

With the resistance $R(\omega)$, capacity $C(\omega)$ and impedance $Z(\omega)$ defined in Section 3.3 the resulting generalization of the Nyquist formula for any non-vanishing frequency looks as $\mathrm{Eq} 47$.

A specific aspect of this somewhat lengthy derivation is that the isolation of the sample (no current flow perpendicular to the boundary cross-section) is explicitly included. However, in both derivations the origin of the fluctuation is the fluctuation of the charge density in the system and the results are identical.

\section{B The Callen-Welton formula.}

Callen and Welton [3] long before Kubo [10] treated linear transport due to an applied potential drop $V$ and related it to the currrent-current correlation. Thereafter they derived the integrated noise spectrum:

$$
\begin{aligned}
\left\langle V^{2}\right\rangle & =\frac{2}{\pi} \int_{0}^{\infty} d \omega\left(\frac{1}{2} \hbar \omega+\hbar \omega\left[e^{\beta \hbar \omega}-1\right]^{-1}\right) R(\omega) \\
& \equiv \frac{1}{\pi} \int_{0}^{\infty} d \omega \hbar \omega \operatorname{coth}\left(\frac{\beta \hbar \omega}{2}\right) R(\omega) .
\end{aligned}
$$


In Ref.[3] no derivation or explicit definition of the noise spectral density is given. Nevertheless the integrand in Eq.83 is usually interpreted as the noise spectral density.

An instant derivation of this spectrum results indeed [4] if one considers the quantum mechanical current fluctuation as the source of the noise, express it by the Kubo formula of the conductivity in its mean-field version (i.e. $V$ is interpreted as the s.c. potential drop) and implicitly to the frequency-dependent resistance

$$
\left(\delta I_{\omega}^{2}\right)^{K}=2 \hbar \omega \operatorname{coth}\left(\frac{\beta \hbar \omega}{2}\right) \frac{1}{R(\omega)} .
$$

Multiplying with $R(\omega)^{2}$ one gets indeed the voltage noise of Ref.[3]

$$
\left(\delta V_{\omega}^{2}\right)^{C W}=2 \hbar \omega \operatorname{coth}\left(\frac{\beta \hbar \omega}{2}\right) R(\omega) .
$$

To our knowledge, this is the first and only direct proof [4] of the CallenWelton spectral density.

It is worth to mention here that the original form Eq.82 suggests a false connection to photons by the presence of the Bose function. As it was shown in Section 5 the photon number noise has nothing to do with the voltage noise.

To conclude, Eq. 85 is valid in the mean-field approximation, while Eq. 47 beyond it. The appearance of the capacitance is a pure Coulomb effect absent in the mean-field approximation.

\section{References}

[1] H. Nyquist, Phys. Rev. 32,110 (1928) https://doi.org/10.1103/PhysRev.32.110

[2] J.B. Johnson, Phys. Rev. 32, 97 (1928) https://doi.org/10.1103/PhysRev.32.97

[3] H.B. Callen, T.A. Welton, Phys. Rev. 8334 (1951) https://doi.org/10.1103/physrev.83.34

[4] L.Bányai, A. Aldea and P. Gartner, Z. Phys. B 58,161 (1985) https://doi.org/10.1007/bf01304270

[5] V.L. Ginzburg and L.O. Pitaevskii, Usp. Fiz. Nauk., 151, 333 (1987) https://doi.org/10.3367/ufnr.0151.198702f.0333

[6] Ladislaus Alexander Bányai, Lectures on Non-Equilibrium Theory of Condensed Matter. Second Edition. World Scientific (2020) https://doi.org/10.1007/978-3-030-37359-7

[7] U. Marini, B. Marconi, A. Puglisi, L. Rondoni and A. Vulpiani, Phys. Rep. 461, Issues 4-6, pp. 111- 195 (2008) https://doi.org/10.1016/j.physrep.2008.02.002 
[8] A. A. Clerk, M. H. Devoret, S. M. Girvin, F. Marquardt, and R. J. Schoelkopf, Rev. Mod. Phys. 82, 1155 (2010)

https://doi.org/10.1103/revmodphys.82.1155

[9] M. Lifshitz, L.P. Pitaevskii, Statistical Phyics. Part 2. Oxford: Pergamon Press (1980)

https://doi.org/10.1016/B978-0-08-057046-4.50008-7

[10] R. Kubo, J. Phys. Soc. Jpn, 12, 570 (1957)

https://doi.org/10.1143/jpsj.12.570

[11] T. Izuyama, Progr. Theor. Phys. 25,964 (1961)

https://doi.org/10.1143/ptp.25.964

[12] D. N. Zubarev, Nonequilibrium statistical thermodynamics. Moscow, Nauka (1971) (English translation: New York, London, Consultants Bureau 1974)

[13] N. Wiener, Norbert (1930 Acta Mathematica. 55, 117 (1930)

https://doi.org/10.1007/bf02546511

[14] A.Khintchine, Mathematische Annalen. 109, (1): 604 (1930) https://doi.org/10.1007/bf01449156

[15] S.R. de Groot and P. Mazur, Non-Equilibrium Thermodynamics, Amsterdam, North-Holland Publ. Co. (1962)

https://doi.org/10.1126/science.140.3563.168

[16] C. G. Darwin, Phil. Mag. ser. 6. 36, 537 (1920)

https://doi.org/10.1080/14786440508636066

[17] L.A. Bányai, Int. J. Theor. Phys. (2021)

https://doi.org/10.1007/s10773-021-04842-9

[18] R.E. Burgess: Electromagnetic fluctuations in an equilibrium plasma, RADIO SCIENCE Journal of Research NBS/USNC-URSI 69D, 381-388 (1964) 